Neurotoxicology

\title{
SUMOylation, aging and autophagy in neurodegeneration
}

\section{Shamini Vijayakumaran and Dean L. Pountney*}

Menzies Health Institute Queensland, School of Medical Science, Griffith University, Gold Coast, Queensland 4222, Australia

\author{
*Corresponding author: Dean L. Pountney \\ Menzies Health Institute Queensland, \\ School of Medical Science, \\ Griffith University, \\ Gold Coast, Queensland 4222, \\ Australia \\ Tel: +61 755527273 \\ Fax: +61 755528908 \\ Email: d.pountney@griffith.edu.au
}




\title{
Highlights
}

- SUMOylation, a post-translation protein modification similar to ubiquitination is discussed in relation to ageing and neurodegeneration

- Modulation of the SUMO pathway is suggested as a therapeutic target against neurodegenerative diseases characterized by protein aggregation

\begin{abstract}
:
Protein homeostasis is essential for the wellbeing of several cellular systems. Posttranslational modifications (PTM) coordinate various pathways in response to abnormal aggregation of proteins in neurodegenerative disease states. In the presence of accumulating misfolded proteins and toxic aggregates, the small ubiquitin-like modifier (SUMO) is associated with various substrates, including chaperones and other recruited factors, for refolding and for clearance via proteolytic systems, such as the ubiquitin-proteasome pathway (UPS), chaperone-mediated autophagy (CMA) and macroautophagy. However, these pathological aggregates are also known to inhibit both the UPS and CMA, further creating a toxic burden on cells. This review suggests that re-routing cytotoxic aggregates towards selective macroautophagy by modulating the SUMO pathway could provide new mechanisms towards neuroprotection.
\end{abstract}

Keywords: neurodegeneration, misfolded protein, post-translational modifications, smallubiquitin-like modifier, autophagy. 
Post-translational modifications in aging and neurodegeneration. In most organisms, ageing is a shift from the fully functional to the deterioration of various physiological processes leading to impaired function and increased vulnerability to death. Ageing is reported to be the major risk factor for the onset and progression of several diseases such as cancer, neurodegeneration and cardiovascular disease (López-Otín et al., 2013; Sen et al., 2016; Soltow et al., 2010). Neurodegenerative diseases diversely comprise of chronic progressive disease states that are characterized by gradual dysfunction and loss of neurons due to the abnormal deposition of misfolded and aggregated proteins in various brain regions (Kovacs et al., 2017; Pratt et al., 2015). Cells lose their ability to cope with the accumulation of oligomeric protein complexes that deteriorate the protein quality control system leading to neurotoxicity and ultimately widespread neurodegeneration (Takalo et al., 2013). These adverse effects that occur within cells are prevented by the process referred to as protein homeostasis or proteostasis. This is orchestrated by several post-translational mechanisms through the aid of protein chaperones, co-chaperones and mediators that fold and/or refold misfolded proteins, maintaining the correct conformation and/or attempting to sequester or degrade toxic soluble misfolded species via aggresomes and autophagy. It has been suggested that cells actively sequester misfolded proteins in chaperone-rich quinary (Q-body) compartments (Alavez, 2017; Escusa-Toret et al., 2013; Roth and Balch, 2013). These misfolded species are then directed to the ubiquitin proteasome system (UPS) and/or the autophagy lysosome pathway (ALP) for degradation so as to prevent neurotoxicity (Manecka et al., 2017). However, the toxic accumulation of protein aggregates has been reported to cause secondary inhibition of the proteasome as well as lysosome system causing a vicious cycle encouraging further aggregation and cell death (Cuervo and Wong, 2014; Schapira et al.). There has been a rising interest in post-translational modification by the small ubiquitin-like modifier (SUMO), known as SUMOylation, as it has been identified as 
one of the key regulators in various cellular mechanisms in age-related diseases (Flotho and Melchior, 2013; Xiao et al., 2015). Neuropathological inclusion bodies found in various neurological conditions have been reported to contain SUMO and/or SUMO target proteins, such as $\alpha$-synuclein, which has been classified as a SUMO substrate. SUMO-1 has also been found to be associated with lysosomes conjugated to Hsp90 in the $\alpha$-synucleopathy, MSA, and in a unilateral PD mouse model suggesting a possible link to chaperone-mediated autophagy (Weetman et al., 2013; Wong et al., 2013). Anderson and co-workers have reviewed the affiliation of SUMO and various SUMO substrates to a range of neurological deficits and cellular stressors such as Parkinson's disease (PD), Alzheimer's disease (AD), polyglutamine (Poly-Q) diseases, oxidative stress, osmotic stress and hypoxic stress (Anderson et al., 2017; Ficulle et al., 2018; Peters et al., 2017; Princz and Tavernarakis, 2017). In this review, we focus on the various cellular responses SUMOylation is linked to in neurodegenerative protein aggregation diseases and the potential of modulating the SUMO pathway to promote neuronal health.

SUMOylation, chaperones, co-chaperones and adaptor proteins. SUMO has been reported to play an important role in DNA repair and telomere maintenance as well as intracellular transport and signalling processes to maintain physiological functions and survival. Peters and colleagues have summarised the various processes and proteins that SUMO regulates in the central nervous system as well as in neurodegeneration (Peters et al., 2017). The SUMO protein covalently modifies acceptor lysine residues of this diverse range of target proteins altering their functional properties through the process called SUMOylation (Iribarren et al., 2015; Krumova and Weishaupt, 2013). SUMO-mediated modulation can occur via the inhibition of the binding site of a protein preventing its interaction with its substrate protein as well as posing as a 'hub' for interaction by recruiting other binding partners. Furthermore, the SUMO modified substrate protein may change in conformation which may lead to 
alterations in its activity or reveal previously exposed binding sites (Vijayakumaran et al., 2015; Wilkinson et al., 2010). SUMO paralogues 1, 2 and 3 are found abundantly in the brains of vertebrates, although SUMO-2 and SUMO-3 are usually collectively referred to as SUMO-2/3 as they are almost identical in sequence. The SUMO-1 isoform is unable to form polySUMO-1 chains like SUMO2/3 isoforms and does not have a large unconjugated cellular pool (Cho et al., 2015; Lee et al., 2014; Vijayakumaran et al., 2015; Yang et al., 2016). Like ubiquitination, the SUMO pathway requires the co-ordinated actions of the SUMO activating E1 enzyme, SUMO-specific conjugating E2 enzyme (Ubc9) and the SUMO ligase (PIAS3) E3 enzyme and their detailed biochemistry is discussed in recent reviews (Eckermann, 2013; Guo and Henley, 2014; Sarge and Park-Sarge, 2011). Regardless of this covalent SUMO modification, the process is easily reversible due to the action of SUMO-specific deSUMOylation enzymes (SENPs). In mammals, there are 6 different SENPs that are known to be regulators of the SUMO pathway (Flotho, 2013; Wilkinson et al., 2010; Yang et al., 2016). As a result, SUMO conjugation is able to act as a reversible switch to facilitate or prevent protein-protein interactions with the substrate protein in response to SUMOylation status. The stability of SENPs, particularly SENP3, is directed by the tumour suppressor protein p19 (arf) promoting the turnover of SENP3 in a UPS-dependent manner. Hsc70interacting protein (CHIP) facilitates the ubiquitination of SENP3 followed by its degradation under normal conditions. SENPs show variable inducibility, cellular and subcellular distribution, thereby fine-tuning SUMO status, with SENP5 showing largely synaptic expression (Mendes et al., 2016; Akiyama et al., 2018). However, it has been reported that under cell stress SENP3 levels are increased, due to its association with the molecular chaperone, heat shock protein 90 (HSP90), which protects it from CHIP-mediated ubiquitination (Guo and Henley, 2014; Yan et al., 2010). 
Various chaperones and co-chaperones such as Hsc70, Hsp70, Hsp90 and Hsp40, that play many crucial roles interact with the SUMO pathway (Wong et al., 2013). It has been proposed that a chaperone-rich Q-body cytoplasmic compartment exists where a decision is made about whether a misfolded protein should undergo degradation or refolding (Roth and Balch, 2013). In addition, various adaptor proteins have been discovered to play essential roles in the UPS and ALP in establishing the fate of misfolded and/or aggregated proteins. Parkin mediates the K-63 poly-ubiquitination associated with aggresome formation (Olzmann, 2008) whereas VCP (P97) disassembles SUMOylated proteins directing them to the UPS (Yamanaka et al., 2012). A balance between VCP and HDAC6, which stimulates aggresome formation, is crucial to determine the fate of ubiquitinated proteins. In VCP mutant cells, aggresome formation was found to be impaired which was counteracted by the expression of HDAC6 showing that they work in unity to ensure proper aggresome formation (Richter-Landsberg and Leyk, 2013). Furthermore, BAG1/BAG3 ratio and CHIP adaptor proteins work together to determine if polyubiquitinated proteins should be directed to the UPS or ALP (Kumar et al., 2007; Shimamoto et al., 2013).

SUMO-1 in neurodegenerative diseases. The disruption of basal SUMOylation in neuronal dysfunction has been implicated in neurodegenerative disorders such as PD, AD and spinocerebellar ataxias (SCAs)(Cho et al., 2015; Matsuzaki et al., 2015). Reports have identified SUMO-1 marking nuclear and cytoplasmic inclusion bodies in familial neuronal intranuclear inclusion disease (NIID), SCA3, Huntington's disease, dementia with Lewy bodies, progressive supranuclear palsy and multiple system atrophy (MSA) (Pountney et al., 2005; Pountney et al., 2003), with punctate SUMO-1 structures in cytoplasmic inclusions assigned to trapped lysosomes. Weetman and co-workers demonstrated that in a rotenonelesion model of PD, increased baseline SUMO-1 expression was observed in aged brain 
compared to young mice in unlesioned hemisphere, whereas, increased SUMOylation occurred in both young and aged mice in the lesioned hemisphere. Moreover, it was also revealed that the co-localisation of SUMO-1 to lysosomes is consistently observed in the affected brain regions of human disease, rodent and cell culture models, providing a link to autophagy in response to aggregation (Weetman et al., 2013). Krumova and coworkers revealed that mutations of the two consensus of the SUMOylation sites led to aggravated $\alpha$ synuclein toxicity reducing the number of dopaminergic neurons in a PD rat model (Krumova et al., 2011). In HD, the pathogenic fragment Huntingtin, was found to be modified by SUMO-1 where it was suggested that the SUMOylated Htt reduced inclusion body formation. However, this did not reduce toxicity but in fact contributed to the disease pathology showing that the reduction of aggregated protein does not always mean a reduction in neurotoxicity (Steffan et al., 2004). Moreover, although both $\alpha$-synuclein and tau have been shown to be SUMOylation targets in vitro, the presence of the SUMOylated species in pathological aggregates is unclear (Wong et al, 2013; Luo et al 2016). Oxidative stress can either increase or decrease SUMOylation. Recently, quercetin was found to increase total SUMOylation and also increase the expression of the oxidative stress master regulator, NRF2, rendering cells more tolerant to glucose/oxygen deprivation (Lee et al, 2016). Furthermore, the overexpression of SUMO in conditions such as brain ischemia and hypoxia, could increase cell survival whereas the knockdown of SUMO expression has proven to be toxic to cells demonstrating evidence that SUMO may play a protective role in cellular responses overcoming stressors such as glucose and oxygen deprivation (Silveirinha et al., 2013; Yang et al., 2016). Indeed, many mitochondrial associated proteins are SUMOylated, such as Drp1 and MFF, thereby influencing mitochondrial fission, the clearance of dysfunctional mitochondria and cell death (Paasch et al., 2018; Guo et al., 2017), and SENP2 knockout that 
blocks Drp1 deSUMOylation was found to induce a neurodegenerative phenotype (Fu et al., 2014).

Modulating SUMOylation as a therapeutic target to combat neurodegeneration. It has been proposed that SUMOylation of target proteins may have direct and/or indirect links to neurodegenerative disease pathology (Vijayakumaran et al., 2015). Various cancer studies have investigated SUMOylated CD44 in tumour cells and showed that inhibiting the SUMO pathway reduced the CD44 expressions repressing invasiveness and tumour growth (Bogachek et al., 2016; Castaño-Miquel et al., 2017; De Andrade et al., 2015). Also, Yun and colleagues demonstrated for the first time that SUMO-1 regulates $\beta$-secretase (BACE1), a protein that recruits $\beta$-amyloid $(\mathrm{A} \beta)$ production in $\mathrm{AD}$ diseased brain. Due to the depletion of SUMO-1, BACE1 proteins were reduced thereby decreasing A $\beta$ levels (Yun et al., 2013). Khodzhigorova and colleagues have reported that the siRNA-mediated knockdown of Ubc9 reduces the accumulation of phosphorylated $S \operatorname{mad} 3$ in experimental fibrosis through the reduction of TGF- $\beta$ signalling suggesting that inhibition of SUMOylation may be a novel therapeutic target (Khodzhigorova et al., 2012). Ubc9 knockdown in neonatal ventricular myocytes led to increase in the autophagy adapter, p62, but reduced L3-II and beclin (Gupta et al., 2015), whereas, Vps34 SUMOylation promotes autophagosome formation (Yang et al, 2013). Various reports have modulated the SUMO pathway using chemical inhibitors. It was first reported that a botanical drug, ginkgolic acid (GA), downregulates protein SUMOylation by blocking the formation of the E1-SUMO intermediate (Fukuda et al., 2009). GA was used in in vitro and in vivo experiments where the treatment of GA impaired the proliferation and restrained tumour growth in pancreatic cancer, showing also that GA had little toxicity on normal cells (Ma et al., 2010). Various studies have indicated that GA treatments have antimigratory and anti-metastatic activity in breast and colon cancer cells (Hamdoun and Efferth, 
2017; Qiao et al., 2017). GA treatment of HEK293 cells led to reduced SUMOylation and decreased phosphorylation, whereas, tau SUMOylation inhibited its degradation (Luo et al, 2014). Kerriamycin B has also been reported as another inhibitor of the E1 enzyme of the SUMO pathway possessing both antimicrobial and antitumor activity (Kumar et al., 2013). Recently, Spectomycin B1 was found to inhibit the E2 enzyme, Ubc9, of the SUMO-1 pathway (Hirohama et al., 2013). As macroautophagy is capable of degrading large structures such as protein aggregates and is reciprocally regulated with CMA, which cannot degrade aggregates, inhibiting SUMOylation of lysosomal Hsp90 and CMA may promote aggregate clearance (Fig 1). Indeed, several inhibitors of Hsp90 have been investigated as therapeutic targets in neurodegeneration. Recently, inhibition of the Hsp90 co-chaperone Aha1 that is activated by SUMOylation was found to block tau aggregation (Shelton et al, 2017). Therefore, targeting the SUMO pathway by utilizing inhibitors such as GA which prevent the formation of the E1-SUMO intermediate, may represent a novel potential therapy for various neurodegenerative diseases.

SUMOylation is involved in several pathways such as protein misfolding and refolding, the UPS and the ALP that modifies the toxic species of aggregated protein through various direct and/or indirect mechanisms. It is important to recognize its associations between various pathways where SUMOylation plays a role in response to accumulating toxic aggregates. However, the demand for a greater understanding of SUMOylation in ageing and neurodegeneration to therapeutically modulate SUMO is inevitable. The benefit of directing toxic aggregates towards selective macroautophagy instead has been suggested, as pathological aggregates have known to inhibit the UPS and CMA. Therefore, we propose that SUMOylation could be specifically modulated to alter the function of various proteins resulting in a protective outcome. 


\section{$\underline{\text { Acknowledgments }}$}

We gratefully acknowledge the financial support of Griffith University and the Menzies Health Institute Queensland. 


\section{References}

Akiyama H, Nakadate K, Sakakibara SI., 2018, Synaptic localization of the SUMOylationregulating protease SENP5 in the adult mouse brain. J Comp Neurol. 526(6):990-1005.

Alavez, S., 2017. Protein Homeostasis and Ageing in C. elegans, in: Olsen, A., Gill, M.S. (Eds.), Ageing: Lessons from C. elegans. Springer International Publishing, Cham, pp. 265-283.

Anderson, D.B., Zanella, C.A., Henley, J.M., Cimarosti, H., 2017. Sumoylation: Implications for Neurodegenerative Diseases, in: Wilson, V.G. (Ed.) SUMO Regulation of Cellular Processes. Springer International Publishing, Cham, pp. 261-281.

Bogachek, M.V., Park, J.M., De Andrade, J.P., Lorenzen, A.W., Kulak, M.V., White, J.R., Gu, V.W., Wu, V.T., Weigel, R.J., 2016. Inhibiting the SUMO Pathway Represses the Cancer Stem Cell Population in Breast and Colorectal Carcinomas. Stem Cell Reports 7(6), 1140-1151.

Castaño-Miquel, L., Mas, A., Teixeira, I., Seguí, J., Perearnau, A., Thampi, B.N., Schapire, A.L., Rodrigo, N., La Verde, G., Manrique, S., Coca, M., Lois, L.M., 2017. SUMOylation Inhibition Mediated by Disruption of SUMO E1-E2 Interactions Confers Plant Susceptibility to Necrotrophic Fungal Pathogens. Molecular Plant 10(5), 709-720.

Cho, S.J., Yun, S.M., Jo, C., Lee, D.H., Choi, K.J., Song, J.C., Park, S.I., Kim, Y.J., Koh, Y.H., 2015. SUMO1 promotes Abeta production via the modulation of autophagy. Autophagy 11(1), 100-112.

Cuervo, A.M., Wong, E., 2014. Chaperone-mediated autophagy: roles in disease and aging. Cell Res 24(1), 92-104.

De Andrade, J.P., Lorenzen, A.W., Bogachek, M.V., Park, J., Robinson, R.A., Lal, G., Weigel, R.J., 2015. Targeting the Small Ubiquitin-Like Modifier Pathway as a Novel Treatment of Anaplastic Thyroid Cancer. Journal of the American College of Surgeons 221(4, Supplement 1), S61.

Eckermann, K., 2013. SUMO and Parkinson's disease. Neuromolecular Med 15(4), 737-759.

Escusa-Toret, S., Vonk, W.I.M., Frydman, J., 2013. Spatial sequestration of misfolded proteins by a dynamic chaperone pathway enhances cellular fitness during stress. Nat Cell Biol 15(10), 1231-1243.

Ficulle, E., Sufian, M.D.S., Tinelli, C., Corbo, M., Feligioni, M., 2018. Aging-related SUMOylation pattern in the cortex and blood plasma of wild type mice. Neuroscience Letters 668, 48-54.

Flotho, A., 2013. Sumoylation: A Regulatory Protein Modification in Health and Disease. Annual review of biochemistry 82(1), 357-385.

Flotho, A., Melchior, F., 2013. Sumoylation: a regulatory protein modification in health and disease. Annu Rev Biochem 82, 357-385.

Fu J, Yu HM, Chiu SY, Mirando AJ, Maruyama EO, Cheng JG, Hsu W., 2014. Disruption of SUMO-specific protease 2 induces mitochondria mediated neurodegeneration. PLoS Genet. 10(10):e1004579.

Fukuda, I., Ito, A., Hirai, G., Nishimura, S., Kawasaki, H., Saitoh, H., Kimura, K., Sodeoka, M., Yoshida, M., 2009. Ginkgolic acid inhibits protein SUMOylation by blocking formation of the E1-SUMO intermediate. Chem Biol 16(2), 133-140.

Guo, C., Henley, J.M., 2014. Wrestling with stress: roles of protein SUMOylation and deSUMOylation in cell stress response. IUBMB life 66(2), 71-77. 
Guo C, Wilkinson KA, Evans AJ, Rubin PP, Henley JM., 2017. SENP3-mediated deSUMOylation of Drp1 facilitates interaction with Mff to promote cell death. Sci Rep. 7:43811.

Hamdoun, S., Efferth, T., 2017. Ginkgolic acids inhibit migration in breast cancer cells by inhibition of NEMO sumoylation and NF-KB activity. Oncotarget 8(21), 35103-35115.

Hirohama, M., Kumar, A., Fukuda, I., Matsuoka, S., Igarashi, Y., Saitoh, H., Takagi, M., Shinya, K., Honda, K., Kondoh, Y., Saito, T., Nakao, Y., Osada, H., Zhang, K.Y.J., Yoshida, M., Ito, A., 2013. Spectomycin B1 as a Novel SUMOylation Inhibitor That Directly Binds to SUMO E2. ACS Chemical Biology.

Iribarren, P.A., Berazategui, M.A., Bayona, J.C., Almeida, I.C., Cazzulo, J.J., Alvarez, V.E., 2015. Different proteomic strategies to identify genuine Small Ubiquitin-like MOdifier targets and their modification sites in Trypanosoma brucei procyclic forms. Cellular microbiology 17(10), 1413-1422.

Khodzhigorova, A., Distler, A., Lang, V., Dees, C., Schneider, H., Beyer, C., Gelse, K., Distler, O., Schett, G., Distler, J.H.W., 2012. Inhibition of sumoylation prevents experimental fibrosis. Annals of the Rheumatic Diseases 71(11), 1904.

Kovacs, G.G., Lee, V.M., Trojanowski, J.Q., 2017. Protein astrogliopathies in human neurodegenerative diseases and aging. Brain Pathology 27(5), 675-690.

Krumova, P., Meulmeester, E., Garrido, M., Tirard, M., Hsiao, H.-H., Bossis, G., Urlaub, H., Zweckstetter, M., Kügler, S., Melchior, F., Bähr, M., Weishaupt, J.H., 2011. Sumoylation inhibits $\alpha$-synuclein aggregation and toxicity. The Journal of Cell Biology 194(1), 49-60.

Krumova, P., Weishaupt, J.H., 2013. Sumoylation in neurodegenerative diseases. Cellular and Molecular Life Sciences 70(12), 2123-2138.

Kumar, A., Ito, A., Hirohama, M., Yoshida, M., Zhang, K.Y., 2013. Identification of sumoylation activating enzyme 1 inhibitors by structure-based virtual screening. Journal of chemical information and modeling 53(4), 809-820.

Kumar, P., Ambasta, R.K., Veereshwarayya, V., Rosen, K.M., Kosik, K.S., Band, H., Mestril, R., Patterson, C., Querfurth, H.W., 2007. CHIP and HSPs interact with $\beta$-APP in a proteasomedependent manner and influence $A \beta$ metabolism. Human molecular genetics $16(7)$, 848864.

Lee, L., Dale, E., Staniszewski, A., Zhang, H., Saeed, F., Sakurai, M., Fa, M., Orozco, I., Michelassi, F., Akpan, N., Lehrer, H., Arancio, O., 2014. Regulation of synaptic plasticity and cognition by SUMO in normal physiology and Alzheimer\&\#39;s disease. 4, 7190.

López-Otín, C., Blasco, M.A., Partridge, L., Serrano, M., Kroemer, G., 2013. The Hallmarks of Aging. Cell 153(6), 1194-1217.

Ma, J.F., Huang, Y., Chen, S.D., Halliday, G., 2010. Immunohistochemical evidence for macroautophagy in neurones and endothelial cells in Alzheimer's disease. Neuropathology and Applied Neurobiology 36(4), 312-319.

Manecka, D.-L., Vanderperre, B., Fon, E.A., Durcan, T.M., 2017. The Neuroprotective Role of Protein Quality Control in Halting the Development of Alpha-Synuclein Pathology. Frontiers in Molecular Neuroscience 10(311).

Matsuzaki, S., Lee, L., Knock, E., Srikumar, T., Sakurai, M., Hazrati, L.-N., Katayama, T., Staniszewski, A., Raught, B., Arancio, O., Fraser, P.E., 2015. SUMO1 Affects Synaptic Function, Spine Density and Memory. 5, 10730.

Mendes AV, Grou CP, Azevedo JE, Pinto MP., 2016. Evaluation of the activity and substrate specificity of the human SENP family of SUMO proteases. Biochim Biophys Acta. 1863(1):139-47. 
Olzmann, J.A., 2008. Parkin-mediated K63-linked polyubiquitination: A signal for targeting misfolded proteins to the aggresome-autophagy pathway. Autophagy 4(1), 85-87.

Paasch F, den Brave F, Psakhye I, Pfander B, Jentsch S., 2018. Failed mitochondrial import and impaired proteostasis trigger SUMOylation of mitochondrial proteins. J Biol Chem. 293(2):599-609.

Peters, M., Wielsch, B., Boltze, J., 2017. The role of SUMOylation in cerebral hypoxia and ischemia. Neurochemistry International 107, 66-77.

Pountney, D.L., Chegini, F., Shen, X., Blumbergs, P.C., Gai, W.P., 2005. SUMO-1 marks subdomains within glial cytoplasmic inclusions of multiple system atrophy. Neuroscience Letters 381(1), 74-79.

Pountney, D.L., Huang, Y., Burns, R.J., Haan, E., Thompson, P.D., Blumbergs, P.C., Gai, W.P., 2003. SUMO-1 marks the nuclear inclusions in familial neuronal intranuclear inclusion disease. Exp Neurol 184(1), 436-446.

Pratt, W.B., Gestwicki, J.E., Osawa, Y., Lieberman, A.P., 2015. Targeting Hsp90/Hsp70-based protein quality control for treatment of adult onset neurodegenerative diseases. Annu Rev Pharmacol Toxicol 55, 353-371.

Princz, A., Tavernarakis, N., 2017. The role of SUMOylation in ageing and senescent decline. Mechanisms of ageing and development 162, 85-90.

Qiao, L., Zheng, J., Jin, X., Wei, G., Wang, G., Sun, X., Li, X., 2017. Ginkgolic acid inhibits the invasiveness of colon cancer cells through AMPK activation. Oncology Letters 14(5), 58315838.

Richter-Landsberg, C., Leyk, J., 2013. Inclusion body formation, macroautophagy, and the role of HDAC6 in neurodegeneration. Acta Neuropathologica 126(6), 793-807.

Roth, D.M., Balch, W.E., 2013. Q-bodies monitor the quinary state of the protein fold. Nat Cell Biol 15(10), 1137-1139.

Sarge, K.D., Park-Sarge, O.-K., 2011. SUMO and its role in human diseases. Int Rev Cell Mol Biol 288(2), 167-183.

Schapira, A.H.V., Olanow, C.W., Greenamyre, J.T., Bezard, E., Slowing of neurodegeneration in Parkinson's disease and Huntington's disease: future therapeutic perspectives. The Lancet 384(9942), 545-555.

Sen, P., Shah, P.P., Nativio, R., Berger, S.L., 2016. Epigenetic Mechanisms of Longevity and Aging. Cell 166(4), 822-839.

Shimamoto, S., Kubota, Y., Yamaguchi, F., Tokumitsu, H., Kobayashi, R., 2013. Ca2+/S100 Proteins Act as Upstream Regulators of the Chaperone-associated Ubiquitin Ligase CHIP (C Terminus of Hsc70-interacting Protein). Journal of Biological Chemistry 288(10), 7158-7168.

Silveirinha, V., Stephens, G.J., Cimarosti, H., 2013. Molecular targets underlying SUMOmediated neuroprotection in brain ischemia. J Neurochem 127(5), 580-591.

Soltow, Q.A., Jones, D.P., Promislow, D.E.L., 2010. A Network Perspective on Metabolism and Aging. Integrative and Comparative Biology 50(5), 844-854.

Steffan, J.S., Agrawal, N., Pallos, J., Rockabrand, E., Trotman, L.C., Slepko, N., Illes, K., Lukacsovich, T., Zhu, Y.Z., Cattaneo, E., Pandolfi, P.P., Thompson, L.M., Marsh, J.L., 2004. SUMO modification of Huntingtin and Huntington's disease pathology. Science (New York, N.Y.) 304(5667), 100-104.

Takalo, M., Salminen, A., Soininen, H., Hiltunen, M., Haapasalo, A., 2013. Protein aggregation and degradation mechanisms in neurodegenerative diseases. American journal of neurodegenerative disease 2(1), 1-14. 
Vijayakumaran, S., Wong, M.B., Antony, H., Pountney, D.L., 2015. Direct and/or Indirect Roles for SUMO in Modulating Alpha-Synuclein Toxicity. Biomolecules 5(3), 1697-1716.

Weetman, J., Wong, M.B., Sharry, S., Rcom-H'cheo-Gauthier, A., Gai, W.P., Meedeniya, A., Pountney, D.L., 2013. Increased SUMO-1 expression in the unilateral rotenone-lesioned mouse model of Parkinson's disease. Neuroscience Letters 544(0), 119-124.

Wilkinson, K.A., Nakamura, Y., Henley, J.M., 2010. Targets and consequences of protein SUMOylation in neurons. Brain Res Rev 64(1), 195-212.

Wong, M., Goodwin, J., Norazit, A., Meedeniya, A.B., Richter-Landsberg, C., Gai, W., Pountney, D., 2013. SUMO-1 is Associated with a Subset of Lysosomes in Glial Protein Aggregate Diseases. Neurotox Res 23(1), 1-21.

Xiao, N., Li, H., Mei, W., Cheng, J., 2015. SUMOylation Attenuates Human $\beta$-Arrestin 2 Inhibition of IL-1R/TRAF6 Signaling. Journal of Biological Chemistry 290(4), 1927-1935.

Yamanaka, K., Sasagawa, Y., Ogura, T., 2012. Recent advances in p97/VCP/Cdc48 cellular functions. Biochimica et Biophysica Acta (BBA) - Molecular Cell Research 1823(1), 130-137.

Yan, S., Sun, X., Xiang, B., Cang, H., Kang, X., Chen, Y., Li, H., Shi, G., Yeh, E.T.H., Wang, B., Wang, X., Yi, J., 2010. Redox regulation of the stability of the SUMO protease SENP3 via interactions with CHIP and Hsp90. The EMBO Journal 29(22), 3773-3786.

Yang, W., Sheng, H., Wang, H., 2016. Targeting the SUMO pathway for neuroprotection in brain ischaemia. Stroke and Vascular Neurology 1(3), 101-107.

Yun, S.-M., Cho, S.-J., Song, J.C., Song, S.Y., Jo, S.A., Jo, C., Yoon, K., Tanzi, R.E., Choi, E.-J., Koh, Y.H., 2013. SUMO1 modulates $A \beta$ generation via BACE1 accumulation. Neurobiology of Aging 34(3), 650-662.

Figure 1. Schematic diagram illustrating the potential mechanisms by which SUMOylation may confront protein misfolding in neurodegenerative diseases, such as Parkinson's disease. A variety of SUMO-modified substrate proteins (Hsc70, Hsp90, Hsp70, HDAC6, VCP) are recruited in response to abnormal protein aggregation in neurodegenerative diseases. Inhibiting the SUMOylation pathway and chaperone-mediated autophagy, may positively upregulate macroautophagy, clearing protein aggregates to alleviate increasing cytotoxicity in neurodegeneration. 


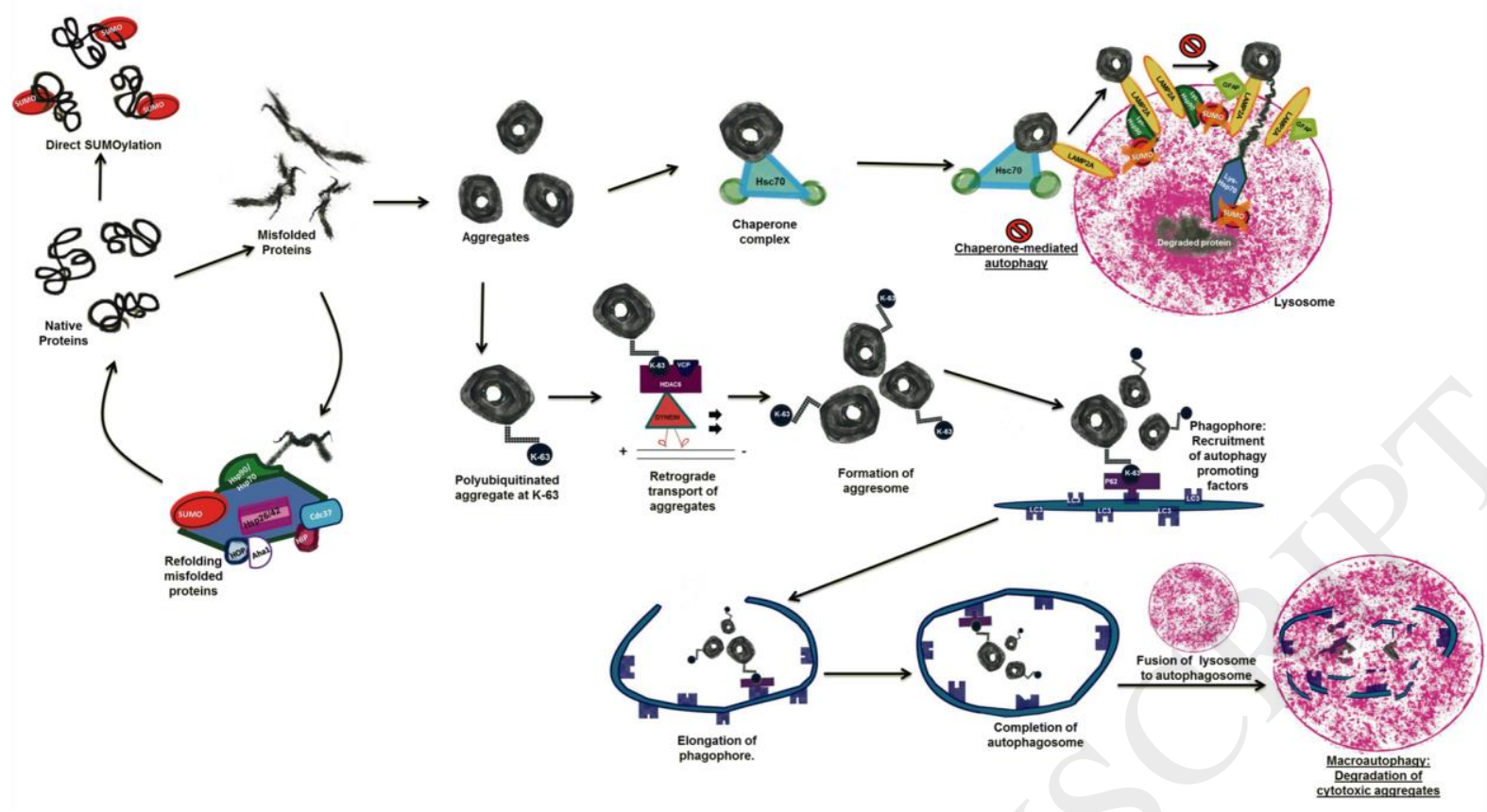




\section{Accepted Manuscript}

Title: SUMOylation, aging and autophagy in neurodegeneration

Authors: Shamini Vijayakumaran, Dean L. Pountney

PII: $\quad$ S0161-813X(18)30056-1

DOI: $\quad$ https://doi.org/10.1016/j.neuro.2018.02.015

Reference: $\quad$ NEUTOX 2303

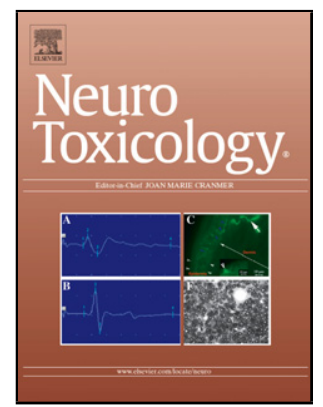

To appear in: NEUTOX

Received date: $\quad 27-10-2017$

Revised date: 22-2-2018

Accepted date: $\quad$ 25-2-2018

Please cite this article as: Vijayakumaran S, Pountney DL, SUMOylation, aging and autophagy in neurodegeneration, Neurotoxicology (2010), https://doi.org/10.1016/j.neuro.2018.02.015

This is a PDF file of an unedited manuscript that has been accepted for publication. As a service to our customers we are providing this early version of the manuscript. The manuscript will undergo copyediting, typesetting, and review of the resulting proof before it is published in its final form. Please note that during the production process errors may be discovered which could affect the content, and all legal disclaimers that apply to the journal pertain. 